\title{
CARBONO ORGÂNICO NO SOLO EM SISTEMAS IRRIGADOS POR ASPERSÃO SOB PLANTIO DIRETO E PREPARO CONVENCIONAL ${ }^{(1)}$
}

\author{
Fabiano Daniel De Bona ${ }^{(2)}$, Cimélio Bayer ${ }^{(3)}$, Homero \\ Bergamaschi $^{(4)}$ \& Jeferson Dieckow ${ }^{(5)}$
}

\begin{abstract}
RESUMO
A irrigação tem efeito sobre a adição de resíduos vegetais e pode afetar a taxa de decomposição da matéria orgânica (MO), sendo a influência dessa prática nos estoques de $\mathrm{C}$ orgânico do solo resultante da magnitude de ambos os efeitos. $O$ objetivo deste estudo foi avaliar o efeito da irrigação por aspersão sobre a dinâmica da MO e sobre os estoques de C orgânico de um Argissolo Vermelho da Depressão Central do RS. O estudo foi baseado em experimento de longa duração (oito anos), realizado em preparo convencional (PC) e plantio direto (PD), em faixas com e sem irrigação por aspersão, em cinco repetições. O solo foi amostrado em quatro camadas até $20,0 \mathrm{~cm}$ de profundidade e o estoque de $\mathrm{C}$ orgânico quantificado. $O$ efeito da irrigação na adição de $\mathrm{C}$ pelas culturas e nas taxas de decomposição da MO do solo e de resíduos culturais foi estimado. A irrigação aumentou a adição de C pelas culturas ( $8 \mathrm{~kg} \mathrm{ha}^{-1}$ ano $^{-1}$ para cada $1 \mathrm{~mm}$ de suplementação hídrica) em ambos os sistemas de manejo, porém isso não se refletiu em aumento no estoque de C orgânico $(0-20,0 \mathrm{~cm})$ pelo fato de a irrigação ter aumentado a taxa de decomposição da MO em 19 \% no solo em PC, e em 15 \% no solo em PD. Os estoques de C orgânico na camada de 0-20,0 cm não diferiram entre os sistemas de preparo de solo, sendo os maiores estoques de $C$ orgânico nas camadas superficiais (0-2,5 e 2,5-5,0 cm) no solo em PD contrabalançados por maiores estoques de $C$ orgânico em subsuperfície $(10,0-20,0 \mathrm{~cm})$ no solo em PC. A taxa de decomposição dos resíduos de aveia preta aumentou com a suplementação hídrica no solo em $\mathrm{PD}$, e os resultados corroboram o efeito da irrigação no aumento da taxa de decomposição da MO no solo. O aumento da taxa de decomposição da MO pela irrigação evidencia
\end{abstract}

\footnotetext{
(1) Parte da Tese de Mestrado do primeiro autor, apresentada ao Programa de Pós-Graduação (PPG) em Ciência do Solo da Universidade Federal do Rio Grande do Sul - UFRGS. Recebido para publicação em abril de 2005 e aprovado em agosto de 2006.

(2) Engenheiro-Agrônomo, Mestre em Ciência do Solo, PPG Solos e Nutrição de Plantas da Escola Superior de Agricultura "Luiz de Queiroz" - ESALQ-USP. Caixa Postal 09, CEP 13418-900 Piracicaba (SP). E-mail: fdbona@esalq.usp.br

(3) Professor Adjunto, Departamento de Solos Universidade Federal do Rio Grande do Sul - UFRGS. Caixa Postal 15100,, CEP 90.001-970 Porto Alegre, RS. E-mail: cimelio.bayer@ufrgs.br

(4) Professor Adjunto, Departamento de Fitotecnia da UFRGS, 90.001-970, C. Postal 15100, Porto Alegre (RS.)

(5) Embrapa Instrumentação Agropecuária, Pós-Doutorando do CNPq. 13560-970, C. Postal 741, São Carlos (SP).
} 
a importância da definição criteriosa de sistemas de manejo de solo em áreas irrigadas em regiões de clima quente, onde se deve buscar a inclusão de espécies com alto potencial de adição de resíduos vegetais, visando contrabalançar o aumento na atividade microbiana com um incremento no aporte de $\mathrm{C}$ fotossintetizado.

Termos de indexação: matéria orgânica, C orgânico, irrigação, manejo de solo, decomposição de resíduos.

\title{
SUMMARY: SOIL ORGANIC CARBON IN SPRINKLER IRRIGATION SYSTEMS UNDER NO-TILL AND CONVENTIONAL TILLAGE
}

\begin{abstract}
The effect of irrigation on soil $C$ stocks is a result of the balance between the effect of this practice on $C$ input through crop residues and of $C$ losses through microbial decomposition of soil organic matter (SOM). This study aimed at assessing the influence of sprinkler irrigation on SOM dynamics and on the total C stocks in a subtropical Acrisol under no-till (NT) and conventional tillage (CT) in a long-term experiment (8 years). Irrigation increased the $C$ addition (about $8 \mathrm{~kg} \mathrm{Cha}^{-1} \mathrm{yr}^{-1}$ per mm precipitation) in both tillage systems, but this was not enough to increase the soil C stocks $(0-20.0 \mathrm{~cm})$ because irrigation also increased the decomposition rate of SOM by $19 \%$ in the CT soil and by $15 \%$ in NT soil. In the top layers (0-2.5 and 2.5-5.0 cm), the Concentration was higher in the NT than in CT soil, while the opposite trend was observed in the deepest layer $(10.0-20.0 \mathrm{~cm})$. Thus, the total $C$ stocks in the whole $0-20.0 \mathrm{~cm}$ layer did not differ between tillage systems. The oat residue decomposition rate in NT soil increased with irrigation, which corroborates the higher SOM decomposition rates estimated for the irrigated soil. Based on these results, it becomes clear that the establishment of a high input cropping system able to counterbalance the higher SOM decomposition rates is a crucial point in the maintenance or improvement of soil $C$ stocks in irrigated tropical and subtropical croplands.
\end{abstract}

Index Terms: organic matter, organic $C$, irrigation, soil management, residue decomposition.

\section{INTRODUÇÃO}

A área sob agricultura irrigada cresce expressivamente no Brasil, correspondendo a, aproximadamente, 3,6 milhões de hectares, dos quais $43 \%$ são irrigados por aspersão (Mittmann, 2004). Embora a resposta das culturas à suplementação hídrica seja bem conhecida (Cunha \& Bergamaschi, 1992), informações referentes às implicações da irrigação sobre os processos biológicos que comandam a decomposição e acúmulo da matéria orgânica (MO) em solos de regiões de clima quente são escassas (Mielniczuk et al., 2003).

A variação anual no estoque de $\mathrm{C}$ orgânico do solo $(d C / d t)$ é resultado do balanço entre a adição de $\mathrm{C}$ fotossintetizado pelas culturas $\left(k_{1} A\right)$ e a perda de $\mathrm{C}$ do solo $\left(k_{2} C\right)$, sendo expressa por $\frac{d C}{d t}=-k_{2} C+k_{1} A$ (Henin \& Dupuis, 1945; Woodruff, 1949; Dalal \& Mayer, 1986; Bayer et al., 2000). Na equação anterior, a variável $C$ corresponde ao estoque atual de $\mathrm{C}$ orgânico do solo, $A$ representa a quantidade anual de $\mathrm{C}$ adicionado ao solo na forma de resíduos, exsudatos radiculares e raízes, $k_{1}$ é o coeficiente de humificação, ou seja, a proporção do $\mathrm{C}$ adicionado anualmente que acumula na MO do solo e $k_{2}$ é a taxa anual de perda de MO por erosão e decomposição microbiana (Dalal $\&$ Mayer, 1986).

O efeito da irrigação sobre o estoque de C orgânico no solo depende de como esta prática influi nos componentes de adição e de perda de C. A irrigação normalmente resulta no aumento da adição de biomassa vegetal produzida pelas culturas $(A)$. Bergonci et al. (2001) verificaram aumento de $53 \%$, ou $5,3 \mathrm{t} \mathrm{ha}^{-1}$, na produção de resíduos de milho decorrente da irrigação por aspersão. O maior rendimento de grãos das culturas decorrente da irrigação, relatado no trabalho de Cunha \& Bergamaschi (1992), também é um indicativo de que o aporte de resíduos culturais é maior em áreas irrigadas.

Entretanto, por aumentar o conteúdo de água no solo, a irrigação pode intensificar a decomposição microbiana da MO $\left(k_{2}\right)$, embora poucos estudos tenham avaliado esse processo. Evidências do efeito favorável da suplementação hídrica na atividade da microbiota foram obtidas por Andrén et al. (1992), os 
quais verificaram maior taxa de decomposição da palha de cevada em sistema irrigado $\left(0,843 \%\right.$ dia $\left.^{-1}\right)$, em comparação ao não irrigado $\left(0,537 \%\right.$ dia $\left.^{-1}\right)$. Esses resultados evidenciam que condições de umidade mais favoráveis para a atividade microbiana podem-se refletir também em aumento da taxa de decomposição da MO em solos sob irrigação.

Em adição ao tipo de solo e à intensidade do déficit hídrico, os sistemas de culturas e de preparo de solo adotados em áreas irrigadas também podem alterar o efeito da irrigação sobre os estoques de C orgânico do solo. Enquanto os sistemas de culturas determinam o potencial de aporte anual de biomassa ao solo, é possível que a utilização de sistemas de preparo com intenso revolvimento do solo favoreça a ocorrência de elevadas taxas de decomposição microbiana da MO do solo, em comparação a sistemas sem revolvimento ou com mínima mobilização (Bayer et al., 2000).

O presente estudo teve por objetivo avaliar o efeito da irrigação por aspersão na dinâmica da MO e nos estoques de $\mathrm{C}$ orgânico de um Argissolo Vermelho manejado em plantio direto e preparo convencional, nas condições de clima subtropical úmido do Sul do Brasil.

\section{MATERIAL E MÉTODOS}

\section{Experimento de campo}

O estudo foi baseado em experimento localizado na Estação Experimental Agronômica da Universidade Federal do Rio Grande do Sul, no município de Eldorado do Sul, RS, na região ecoclimática da Depressão Central. As coordenadas geográficas do local são $30^{\circ} 05^{\prime} \mathrm{S}$ e $51^{\circ} 40^{\prime} \mathrm{W}$, e a altitude atinge 40 m (Bergamaschi et al., 2003). O clima da região é subtropical úmido de verão quente, Cfa, segundo a classificação climática de Köppen. O solo é um Argissolo Vermelho distrófico típico (Embrapa, 1999), com $543 \mathrm{~g} \mathrm{~kg}^{-1}$ de areia, $271 \mathrm{~g} \mathrm{~kg}^{-1}$ de silte e $186 \mathrm{~g} \mathrm{~kg}^{-1}$ de argila, e o relevo é plano.

O experimento foi instalado em 1995, em área mantida por, aproximadamente, três anos sob vegetação espontânea após uso em pesquisa com forrageiras. No início do experimento, a camada de 0-20,0 cm do solo apresentou as seguintes características químicas: $21,0 \mathrm{~g} \mathrm{~kg}^{-1}$ de matéria orgânica; pH 5,9 em água $(1: 2,5)$; teores de $\mathrm{Al}, \mathrm{Ca}$, $\mathrm{Mg}$ e $\mathrm{K}$ trocáveis de $0,0,2,4,0,7$, e $0,3 \mathrm{cmol}_{\mathrm{c}} \mathrm{kg}^{-1}$, respectivamente; e $8,5 \mathrm{mg} \mathrm{kg}^{-1}$ de $\mathrm{P}$ (Mehlich-1). Apesar de não ter sido verificada acidez no solo, na instalação do experimento, foram aplicados $4 \mathrm{t} \mathrm{ha}^{-1}$ de calcário como fonte de $\mathrm{Ca}$ e $\mathrm{Mg}$ com vistas em corrigir o solo para iniciar o plantio direto, além de $180 \mathrm{~kg} \mathrm{ha}^{-1}$ de $\mathrm{P}_{2} \mathrm{O}_{5}$ e 140 de $\mathrm{K}_{2} \mathrm{O}$, os quais foram incorporados na camada de $0-20,0 \mathrm{~cm}$, mediante lavrações e gradagens. O experimento englobou dois sistemas de preparo do solo (plantio direto - PD e preparo convencional - PC) e dois níveis de irrigação por aspersão (irrigado e não irrigado). Os sistemas de preparo foram aplicados em duas subáreas de 22,5 m de largura e 75 m de extensão. Uma linha central de aspersores localizada entre essas subáreas irriga aproximadamente $12 \mathrm{~m}$ de cada uma delas, correspondente ao raio de alcance máximo dos aspersores, com vistas em obter uma faixa irrigada e outra não irrigada. A extensão de cada faixa foi dividida em cinco repetições.

O sistema de culturas foi baseado na sucessão aveia preta (Avena strigosa) + ervilhaca (Vicia sativa), no inverno, e milho (Zea mays), no verão, durante o período 1995-2002. O sistema PD consistiu na ausência total de práticas que mobilizem o solo, excetuando o movimento do solo na linha de semeadura provocado pela ação mecânica do sulcador da máquina semeadora. $\mathrm{O}$ sistema $\mathrm{PC}$ consistiu de uma aração e duas gradagens no final de setembro ou início de outubro, para posterior semeadura do milho. Salienta-se que o consórcio aveia + ervilhaca sempre foi instalado em sistema PD em toda a área experimental. No ano de 2003, foi introduzida a soja no sistema de culturas, substituindo o milho na safra de verão. Detalhes dos tratos culturais e da adubação das culturas são descritos em Dalmago (2004).

A necessidade de irrigação foi monitorada por meio de um conjunto de tensiômetros de coluna de $\mathrm{Hg}$, instalados até $70,0 \mathrm{~cm}$ de profundidade. A lâmina de água aplicada era definida com base nos dados de evapotranspiração, obtidos num lisímetro de pesagem, de $5,1 \mathrm{~m}^{2}$, e localizado no centro de uma parcela homogênea de 0,54 ha adjacente ao experimento. Dados meteorológicos (radiação solar, temperatura do ar, evaporação do tanque classe A, umidade relativa, velocidade do vento e precipitação pluvial) foram coletados em uma estação automática Campbell, localizada a cerca de $50 \mathrm{~m}$ do lisímetro. O uso da irrigação ficou restrito ao ciclo das culturas de verão (milho e soja).

\section{Amostragem e análise do solo e cálculo dos estoques de $\mathrm{C}$ orgânico}

Amostras de solo das camadas de 0-2,5, 2,5-5,0, 5,0-10,0 e 10,0-20,0 cm das parcelas de PD e PC, com e sem irrigação, nas cinco repetições, foram coletadas com auxílio de espátulas numa área retangular de 20,0 x 50,0 cm, em outubro de 2003, antes da rolagem da cobertura de inverno (aveia + ervilhaca) e semeadura da soja. Os resíduos superficiais em cada ponto de coleta foram removidos antes da amostragem para evitar contaminação. As amostras de solo foram secas ao ar, moídas em moinho de bolas até passar em peneira com malha de $2 \mathrm{~mm}$ e armazenadas em potes plásticos. Uma subamostra foi moída em almofariz de ágata e analisada por combustão seca, quanto ao teor de C orgânico, em analisador Shimadzu TOC-V CSH. 
Os estoques de $\mathrm{C}$ orgânico foram calculados pelos métodos de camada e de massa de solo equivalentes. O método da camada equivalente leva em consideração a espessura da camada e a densidade do solo (Bayer et al., 2000), enquanto o método da massa equivalente de solo utiliza, como referência, a massa de solo de um tratamento, a qual é tomada como base para o cálculo do estoque em todos os demais tratamentos (Ellert \& Bettany, 1995).

No presente estudo, foram utilizadas, como referência, as massas de solo das camadas correspondentes de uma área sob campo nativo adjacente ao experimento (De Bona, 2004), a qual representa a condição original do solo (Quadro 1).

\section{Estimativa da taxa de decomposição da MO do solo}

Considerando o estoque de $\mathrm{C}$ orgânico da camada de 0-20,0 cm como sendo o estoque estável de C orgânico $(C e)$, a taxa de decomposição da $\mathrm{MO}\left(k_{2}\right)$ em cada tratamento foi calculada a partir da equação $k_{2}=k_{1}$.A/Ce, rearranjada a partir da equação original $C e=k_{1} \cdot A / k_{2}$ (Woodruff, 1949; Dalal \& Mayer, 1986). As adições de fitomassa pelas culturas de inverno foram quantificadas em três avaliações anuais, sendo utilizados os valores médios. As adições de fitomassa pela parte aérea do milho foram calculadas com base no rendimento anual de grãos e no índice de colheita aparente (grãos/grãos + parte aérea) dos cultivares utilizados no decorrer do período experimental. A partir dos dados de fitomassa da parte aérea, as adições anuais de $\mathrm{C}$ ao solo (A) foram calculadas, considerando uma concentração de C na fitomassa de $40 \%$ e uma contribuição do sistema radicular equivalente a $30 \%$ do $\mathrm{C}$ adicionado pela parte aérea das culturas (Bayer et al., 2000). Para conversão do $\mathrm{C}$ adicionado em $\mathrm{C}$ incorporado na MO do solo, utilizaram-se valores de $k_{1}$ equivalentes a 0,146 , para o $\mathrm{PD}$, e a 0,148 , para o PC (Lovato et al., 2005).

Quadro 1. Densidade dos solos sob preparo convencional (PC), plantio direto (PD) e campo nativo, utilizada, como referência, no cálculo dos estoques de $C$ orgânico em camada e massa equivalentes de solo

\begin{tabular}{cccc}
\hline Profundidade & PC & PD & Campo nativo \\
\hline $\mathrm{cm}$ & & $\mathrm{g} \mathrm{cm}^{-3}$ & \\
$0,0-5,0$ & 1,74 & 1,63 & 1,47 \\
$5,0-10,0$ & 1,60 & 1,61 & 1,69 \\
$10,0-20,0$ & 1,58 & 1,65 & 1,75 \\
& & & \\
\hline
\end{tabular}

Fonte: Dalmago (2004) e De Bona (2005).

\section{Determinação das taxas de decomposição dos resíduos culturais de aveia preta}

Visando obter informações adicionais do efeito da irrigação sobre a atividade da microbiota decompositora, avaliou-se a decomposição de resíduos de aveia preta, utilizando, para tal, sacos de decomposição nos tratamentos de preparo de solo (PC e PD) e de irrigação (com e sem irrigação). Os sacos de decomposição apresentavam dimensões internas de 20,0 x 20,0 cm e foram preenchidos com $16 \mathrm{~g}$ de resíduos secos ao ar (7,5\% de umidade) e grosseiramente picados (aproximadamente 5,0 cm), correspondendo a 3,7 t ha-1 de MS.

Os sacos de decomposição foram distribuídos no campo logo após a semeadura da soja, na segunda quinzena de novembro de 2003, sendo recolhidos ao longo do tempo até à metade do mês de abril de 2004 (maturação fisiológica da cultura). No solo em PC, os sacos de decomposição foram enterrados a aproximadamente $8,0 \mathrm{~cm}$ de profundidade, enquanto, no sistema $\mathrm{PD}$, foram dispostos sobre a superfície do solo. As coletas foram realizadas aos 10, 21, 34, 52, 73, 94 e 142 dias da colocação no campo. Após a coleta, as amostras foram secas em estufa a $60^{\circ} \mathrm{C}$ por $48 \mathrm{~h}$, pesadas e moídas. Uma subamostra foi utilizada para quantificação do conteúdo de cinzas após queima em mufla SANCHIS ${ }^{\circledR}$ a $550{ }^{\circ} \mathrm{C}$ por 5 h. O C orgânico das amostras foi determinado por combustão seca em analisador SHIMADZU TOC-V CSH. O teor de C das amostras foi corrigido de acordo com a quantidade de solo no interior dos sacos de decomposição, conforme proposto por Christensen (1985).

Equações de regressão do tipo exponencial simples (Wieder \& Lang, 1982) foram ajustadas para obter as taxas de decomposição do resíduo de aveia preta $(k)$ nos diferentes tratamentos de preparo de solo e de irrigação, com auxílio do programa computacional Sigma Plot ${ }^{\circledR}$.

\section{Análise estatística}

Os resultados de concentração e estoque de C orgânico no solo foram submetidos à análise da variância, de acordo com o delineamento experimental em faixas, utilizando o aplicativo estatístico System Analysis Statistic (SAS, 1996). A diferença entre médias foi avaliada pelo teste de Tukey $(P<0,05)$.

\section{RESULTADOS E DISCUSSÃO}

\section{Adição de C pelas culturas}

A irrigação aumentou a adição de $\mathrm{C}$ pelas culturas em $19 \%$ (1,6 t ha-1 ano-1) em relação ao sistema não irrigado (Quadro 2), a qual não foi afetada pelos sistemas de preparo de solo. $\mathrm{O}$ aumento da adição de $\mathrm{C}$ foi atribuído a uma redução média de 46 \% do déficit 
hídrico no sistema irrigado (112 mm) em comparação ao não irrigado $(208 \mathrm{~mm})$ durante o ciclo de crescimento e desenvolvimento das plantas (Quadro 2). A irrigação não tornou nulo o déficit hídrico, uma vez que a estimativa do balanço hídrico considera uma capacidade de armazenamento de água disponível (CAD) no solo fixa durante todo o ciclo da cultura (neste estudo $\mathrm{CAD}=75 \mathrm{~mm}$ ).

Considerando a suplementação hídrica média de $195 \mathrm{~mm}$ ano $^{-1}$, estima-se que cada $1 \mathrm{~mm}$ de água fornecido pela irrigação aumentou a adição de $\mathrm{C}$ em cerca de $8 \mathrm{~kg} \mathrm{ha}^{-1}$ ano $^{-1}$. Após oito anos, a área irrigada recebeu aproximadamente $12,7 \mathrm{t} \mathrm{ha}^{-1}$ de $\mathrm{C}$ na forma de resíduos vegetais a mais que a área não irrigada (Quadro 2).

\section{Concentrações e estoques de C orgânico no solo}

As concentrações de $\mathrm{C}$ orgânico nas camadas superficiais $(0-2,5$ e 2,5-5,0 cm) do solo sob PD foram superiores às do solo em PC (Figura 1a, 1b), o que é coerente com o fato de que no $\mathrm{PD}$ os resíduos culturais são mantidos na superfície do solo (Reicosky et al., 1995; Bayer \& Mielniczuk, 1997; Bayer et al., 2000). Por outro lado, na camada de 10,0-20,0 cm, os teores de C orgânico foram superiores no solo sob PC, sendo esse resultado atribuído à incorporação anual dos resíduos em camadas subsuperficiais do solo pelas operações de lavração e gradagem (Figura 1a, 1b).

Em relação aos estoques de $\mathrm{C}$ orgânico, os métodos de cálculo de camada equivalente (Bayer et al., 2000) e de massa equivalente (Ellert \& Bettany, 1995) resultaram em valores muito similares (Quadro 3), graças à pequena variação na densidade do solo entre os sistemas de preparo (Quadro 1). Por ser teoricamente o método mais correto, a discussão dos efeitos dos sistemas de manejo nos estoques de C orgânico será baseada nos resultados obtidos pelo método da massa equivalente de solo.

Os maiores estoques de C orgânico nas camadas superficiais do solo em PD foram compensados pelos maiores estoques de $\mathrm{C}$ orgânico em subsuperfície no solo em PC (Quadro 3). Por esse motivo, os estoques totais de $\mathrm{C}$ na camada de $0-20,0 \mathrm{~cm}$ não diferiram entre os sistemas de preparo (Quadro 3). Apesar de o acúmulo de $\mathrm{C}$ em subsuperfície no solo sob PC compensar o acúmulo de $\mathrm{C}$ nas camadas superficiais do solo sob $\mathrm{PD}$, de forma que o estoque total de $\mathrm{C}$ nos dois sistemas seja semelhante, o acúmulo superficial de $\mathrm{C}$ no solo sob PD ainda é preferível ao acúmulo em subsuperfície no solo sob PC. A MO é fundamental na ciclagem de nutrientes, na estabilidade da estrutura do solo, nos fluxos de $\mathrm{O}_{2}$ e água, na complexação de metais e na atividade da biota (Reicosky et al., 1995). Por conseguinte, sua localização em camadas superficiais, onde se localiza grande parte do sistema radicular (Merten \& Mielniczuk, 1991), pode ser considerado um aspecto benéfico aos cultivos agrícolas.

A irrigação diminuiu a concentração de C orgânico na camada de 0-2,5 cm (Figura 1c, $1 \mathrm{~d}$ ), um resultado

Quadro 2. Déficit hídrico, suplementação hídrica e adição anual de C ao solo nas áreas irrigadas e não irrigadas por aspersão no período experimental 1995-2002. Médias dos sistemas de preparo convencional e plantio direto

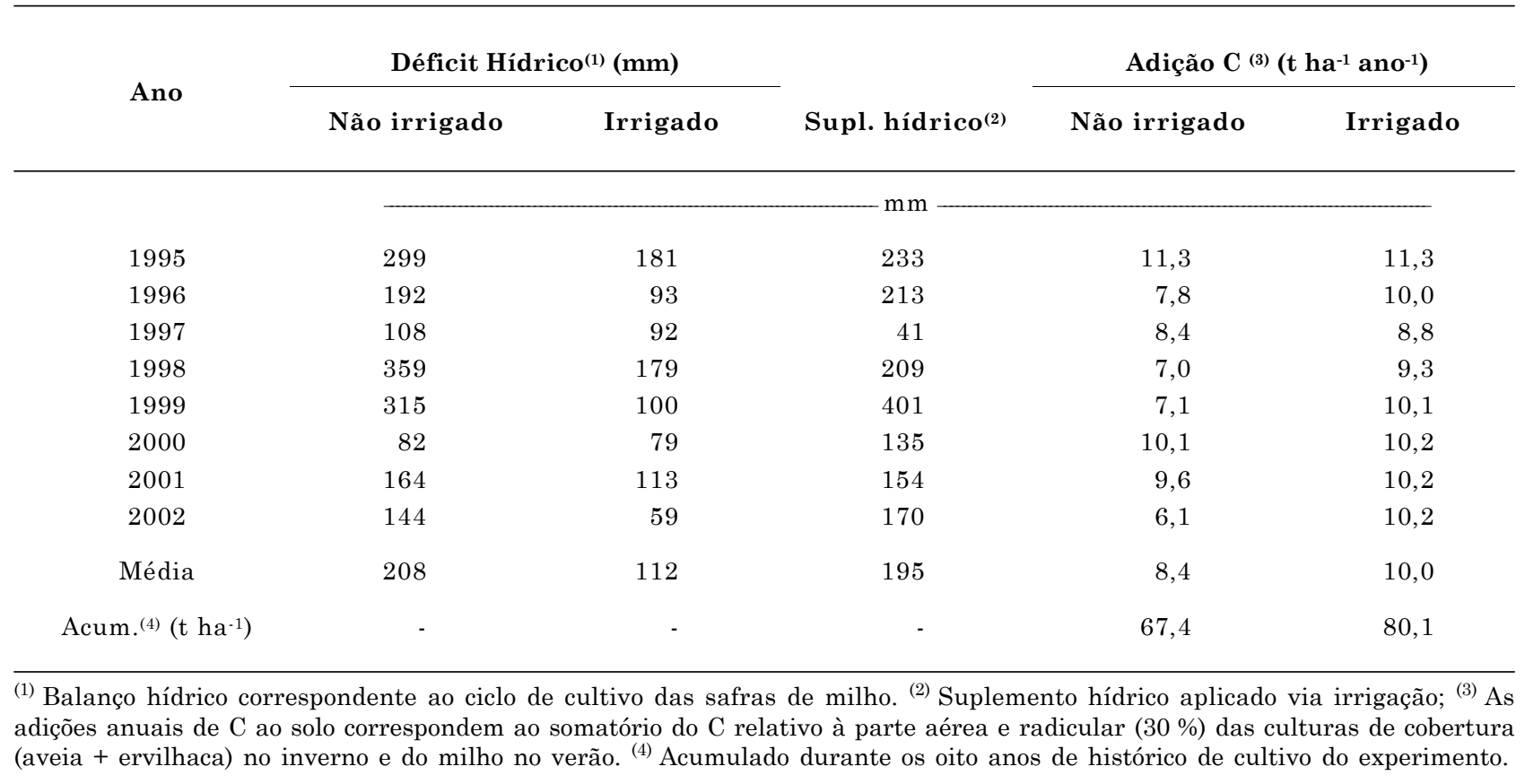




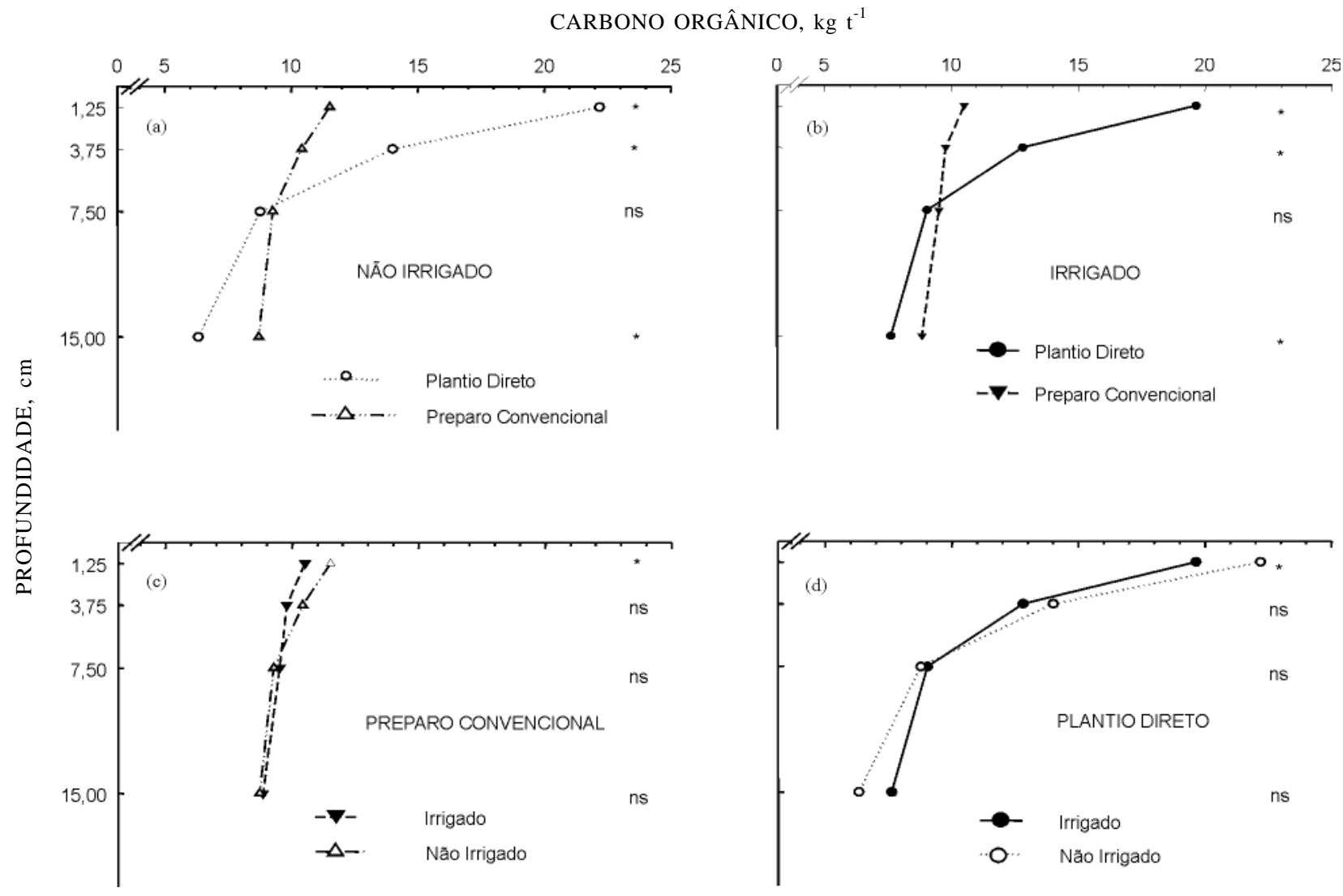

Figura 1. Concentração de C orgânico em Argissolo Vermelho, considerando o sistema de preparo de solo nas áreas (a) não irrigada e (b) irrigada e a irrigação no solo em (c) preparo convencional e (d) plantio direto.

negativo para a qualidade do solo e, em princípio, contraditório ao fato de a irrigação aumentar a adição de resíduos culturais (Quadro 2). Por sua vez, a irrigação não teve efeito nos estoques de $\mathrm{C}$ orgânico em nenhuma camada de solo em comparação ao sistema não irrigado (Quadro 3), apesar do seu efeito expressivo no aporte anual de resíduos vegetais. Esse resultado foi relacionado com o efeito da irrigação no aumento da taxa de decomposição da MO do solo.

\section{Taxas de decomposição da MO do solo}

A taxa média de decomposição da $\mathrm{MO}$ no solo sob PC $\left(0,0438\right.$ ano $\left.^{-1}\right)$ foi semelhante à taxa média de decomposição no solo sob PD $\left(0,0415\right.$ ano $\left.^{-1}\right)$ (Figura 2), demonstrando que o PD não teve efeito significativo na diminuição da taxa de decomposição da MO como inicialmente esperado. Lovato et al. (2005), também trabalhando num Argissolo Vermelho $\left(220 \mathrm{~g} \mathrm{~kg}^{-1} \mathrm{de}\right.$ argila) sob irrigação, verificaram que a taxa de decomposição da $\mathrm{MO}$ do solo sob PC $\left(0,035\right.$ ano $\left.^{-1}\right)$ foi quase o dobro daquela no solo sob PD $\left(0,020 \mathrm{ano}^{-1}\right)$. A alta taxa de decomposição da MO estimada no solo em PD pode estar associada ao fato de o solo em estudo apresentar-se em estádio intermediário de degradação física, evidenciando que a melhoria da estrutura do solo e a conseqüente oclusão da $\mathrm{MO}$ em agregados estáveis não estão sendo expressos em todo seu potencial, limitando o acúmulo de MO no solo em PD. Outra hipótese que não deve ser descartada é que a taxa de decomposição da MO no solo em PD esteja sendo superestimada pelo fato de o estoque de C orgânico no solo sob esse sistema de manejo conservacionista continue acumulando lentamente e não atingiu um estoque estável (Mann, 1986), como foi assumido para calcular a taxa de decomposição a partir da equação $k_{2}=k_{1} \cdot A / C e$.

A irrigação aumentou a taxa de decomposição da MO em $19 \%$ no solo sob PC $\left(0,0400\right.$ ano $^{-1}$ para 0,0477 ano $\left.^{-1}\right)$ e em $15 \%$ no solo sob PD $(0,0386$ para 0,0445 ano $^{-1}$ ) (Figura 2). Este aumento deveu-se à maior disponibilidade de água para a microbiota heterotrófica. Bloem et al. (1992) e Orchard \& Cook (1983) observaram que aumentos relativamente pequenos no potencial de água eram acompanhados de aumentos da atividade microbiana. Fica evidente, portanto, que a maior adição de resíduos culturais no solo irrigado não foi suficiente para aumentar o estoque de $\mathrm{C}$ no solo, por ter sido seu efeito contrabalançado pela maior taxa de decomposição da $\mathrm{MO}$, de forma que o efeito líquido da irrigação sobre os estoques de $\mathrm{C}$ orgânico no solo foi nulo. 
Quadro 3. Estoques de C orgânico calculados em camada equivalente de solo e em massa equivalente de solo, nos sistemas plantio direto (PD) e preparo convencional (PC), com (Irrig.) e sem irrigação (NIrrig.) por aspersão

\begin{tabular}{|c|c|c|c|c|c|c|c|}
\hline \multirow{2}{*}{ Sistema } & \multicolumn{4}{|c|}{ Camada equivalente de solo } & \multicolumn{3}{|c|}{ Massa equivalente de solo } \\
\hline & \multirow{2}{*}{$\begin{array}{c}\text { Camada } \\
\text { cm }\end{array}$} & \multirow{2}{*}{$\begin{array}{c}\text { Massa }^{(1)} \\
\text { t ha }^{-1}\end{array}$} & \multicolumn{2}{|c|}{ Carbono } & \multirow{2}{*}{$\begin{array}{c}\text { Camada }^{(2)} \\
\mathrm{cm}\end{array}$} & \multirow{2}{*}{$\begin{array}{c}\operatorname{Massa}^{(3)} \\
-\mathrm{t} \mathrm{h}\end{array}$} & \multirow[t]{2}{*}{ Carbono $^{(4)}$} \\
\hline & & & $\operatorname{kg~t}^{-1}$ & $\mathrm{t} \mathrm{ha}^{-1}$ & & & \\
\hline \multirow[t]{5}{*}{ PC N-Irrig. } & $0-2,5$ & 435 & 11,5 & 5,0 & $0-2,1$ & 368 & 4,2 a B \\
\hline & $2,5-5,0$ & 435 & 10,4 & 4,5 & $2,1-4,2$ & 368 & 3,8 a B \\
\hline & $5,0-10,0$ & 802 & 9,3 & 7,4 & $4,2-9,5$ & 846 & 7,9 а $\mathrm{A}$ \\
\hline & $10,0-20,0$ & 1581 & 8,7 & 13,8 & $9,5-20,6$ & 1.748 & 15,2 а $\mathrm{A}$ \\
\hline & $0-20,0$ & & & 30,7 & $0-20,6$ & & 31,1 a $\mathrm{A}$ \\
\hline \multirow[t]{5}{*}{ PC Irrig. } & $0-2,5$ & 435 & 10,5 & 4,6 & $0-2,1$ & 368 & 3,9 a B \\
\hline & $2,5-5,0$ & 435 & 9,8 & 4,3 & $2,1-4,2$ & 368 & 3,6 a $\mathrm{A}$ \\
\hline & $5,0-10,0$ & 802 & 9,5 & 7,6 & $4,2-9,5$ & 846 & 8,0 a $\mathrm{A}$ \\
\hline & $10,0-20,0$ & 1581 & 8,9 & 14,0 & $9,5-20,6$ & 1.748 & 15,5 a $\mathrm{A}$ \\
\hline & $0-20,0$ & & & 30,5 & $0-20,6$ & & 31,0 a $\mathrm{A}$ \\
\hline \multirow{5}{*}{ PD N-Irrig. } & $0-2,5$ & 407 & 22,2 & 9,0 & $0-2,3$ & 368 & 8,2 a $A$ \\
\hline & $2,5-5,0$ & 407 & 14,0 & 5,7 & $2,3-4,5$ & 368 & 5,2 a $A$ \\
\hline & $5,0-10,0$ & 806 & 8,8 & 7,1 & $4,5-9,8$ & 846 & 7,4 a B \\
\hline & $10,0-20,0$ & 1652 & 6,3 & 10,4 & $9,8-20,4$ & 1.748 & 11,0 a B \\
\hline & $0-20,0$ & & & 32,2 & $0-20,4$ & & 31,8 a A \\
\hline \multirow[t]{5}{*}{ PD Irrig. } & $0-2,5$ & 407 & 19,6 & 8,0 & $0-2,3$ & 368 & 7,2 a $\mathrm{A}$ \\
\hline & $2,5-5,0$ & 407 & 12,8 & 5,2 & $2,3-4,5$ & 368 & 4,7 a $\mathrm{A}$ \\
\hline & $5,0-10,0$ & 806 & 9,0 & 7,3 & $4,5-9,8$ & 846 & 7,6 а $\mathrm{A}$ \\
\hline & $10,0-20,0$ & 1652 & 7,6 & 12,6 & $9,8-20,4$ & 1.748 & 13,3 a $\mathrm{B}$ \\
\hline & $0-20,0$ & & & 33,1 & $0-20,4$ & & 32,8 a $\mathrm{A}$ \\
\hline$\Delta_{0-20}$ Irrigação $\mathrm{PD}^{(5)}$ & & & & $-0,9$ & & & $-1,0$ \\
\hline$\Delta_{0-20}$ Irrigação PC(6) & & & & 0,2 & & & 0,1 \\
\hline$\Delta_{0-20}$ Preparos s/Irrigação(7) & & & & 1,5 & & & 0,7 \\
\hline$\Delta_{0-20}$ Preparos c/Irrigação(8) & & & & 2,6 & & & 1,8 \\
\hline
\end{tabular}

(1) Massa de solo de cada tratamento, de acordo com os valores de densidade apresentados no quadro 1. ${ }^{(2)}$ Limites teóricos de cada camada que contém a mesma massa de solo do campo nativo (referência). ${ }^{(3)}$ Massa de solo do campo nativo (referência), de acordo com os valores de densidade apresentados no quadro $1 .{ }^{(4)}$ Letras minúsculas comparam os níveis de irrigação dentro do mesmo sistema de preparo. Letras maiúsculas comparam os sistemas de preparo dentro do mesmo nível de irrigação. Médias seguidas de letras diferentes diferem pelo teste de Tukey $(P<0,05) .{ }^{(5)} \Delta_{0-20}$ Irrigação PD = PD não irrigado - PD irrigado. ${ }^{(6)} \Delta_{0-20}$ Irrigação PC = PC não irrigado $-\mathrm{PC}$ irrigado. ${ }^{(7)} \Delta_{0-20}$ Preparos s/Irrigação $=$ PD não irrigado $-\mathrm{PC}$ não irrigado. ${ }^{\left({ }^{(8)}\right.} \Delta_{0-20}$ Preparos c/Irrigação $=$ PD irrigado - PC irrigado.

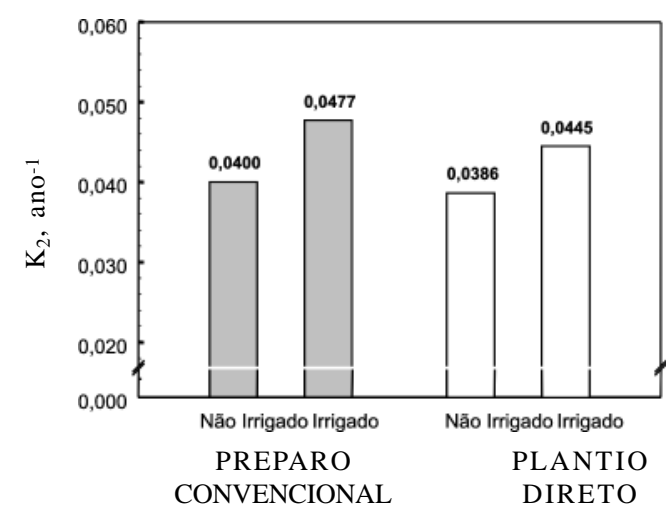

Figura 2. Taxas de decomposição da matéria orgânica do solo $\left(k_{2}\right)$ estimadas para o solo submetido aos sistemas de manejo, preparo convencional e plantio direto, com e sem irrigação por aspersão.

\section{Taxa de decomposição dos resíduos culturais}

O efeito da irrigação sobre a taxa de decomposição da MO do solo é corroborado pelo aumento verificado na taxa de decomposição dos resíduos de aveia preta $(k)$, principalmente no sistema $\mathrm{PD}$, em que a taxa de decomposição na área irrigada foi de 0,0106\% dia-1, enquanto, na não irrigada, foi de $0,0075 \% \mathrm{dia}^{-1}$ (Figura 3b, 3c). Isso reforça a hipótese de que a atividade da microbiota decompositora foi intensificada com a diminuição do déficit hídrico (Figura 3a).

No solo sob PC, não se verificou a influência da irrigação sobre a taxa de decomposição dos resíduos vegetais (Figura 3b, 3c). Provavelmente isso se deve ao fato de que apenas uma fração da lâmina de água adicionada por evento de irrigação tenha atingido a profundidade na qual se encontravam os resíduos 

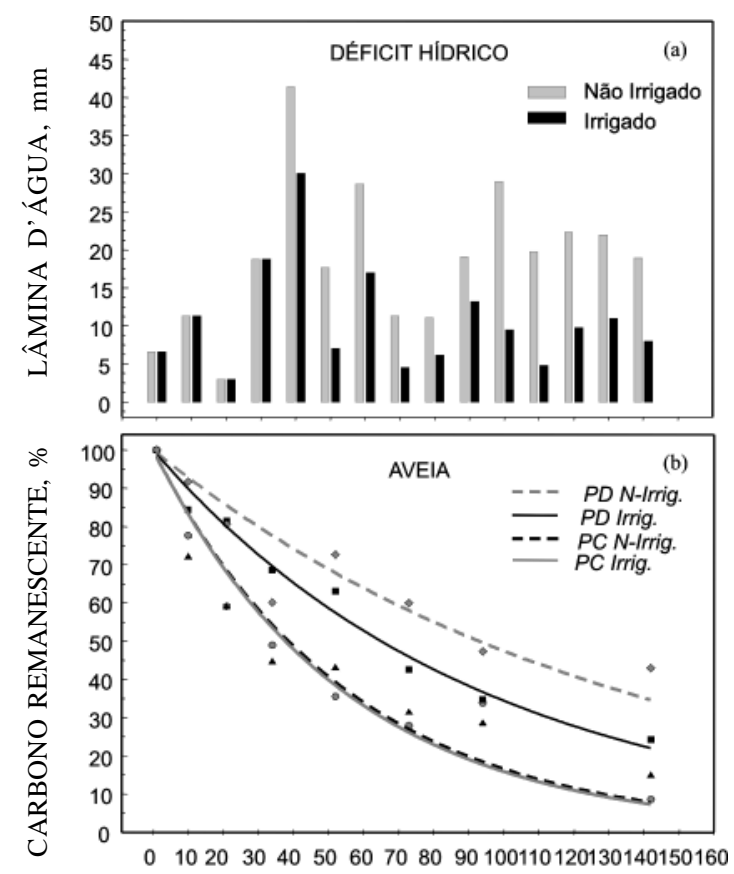

TEMPO, DIA APÓS COLOCAÇÃO NO CAMPO

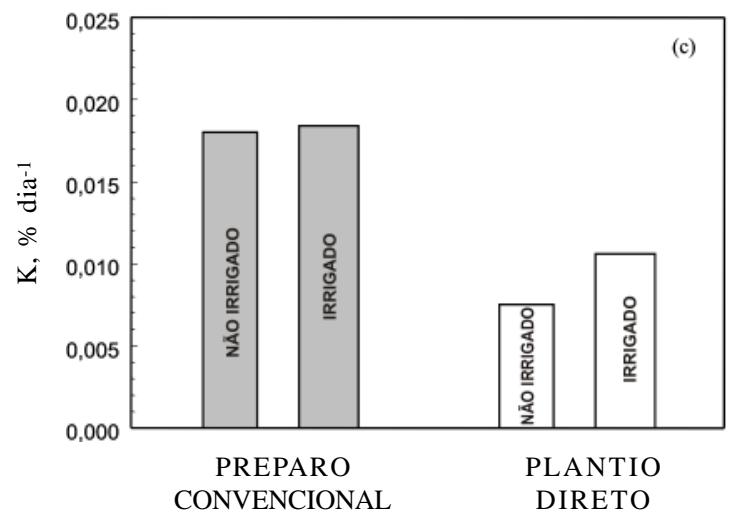

Figura 3. Déficit hídrico estimado para cultura da soja (a), carbono remanescente nos sacos de decomposição (b) e taxas diárias de decomposição dos resíduos culturais de aveia $(k)$ (c) no solo cultivado sob plantio direto (PD) e preparo convencional (PC), com (Irrig.) e sem irrigação (N-Irrig.) por aspersão.

vegetais $(8,0 \mathrm{~cm})$, levando em consideração que a lâmina máxima de água aplicada não foi maior que $30 \mathrm{~mm}$. Além disso, o empoçamento de água verificado na superfície do solo em PC nos eventos de irrigação reflete uma infiltração menor de água nesse solo (Dalmago, 2004).

\section{CONCLUSÕES}

1. O aumento da adição de resíduos vegetais pela irrigação foi contrabalançado pelo aumento das taxas de decomposição da matéria orgânica do solo, sendo nulo o efeito dessa prática nos estoques de C orgânico do solo. O efeito da irrigação na taxa de decomposição microbiana da matéria orgânica do solo foi corroborado pelas maiores taxas de decomposição dos resíduos vegetais em condições de suplementação hídrica.

2. O sistema de preparo do solo não teve efeito no estoque total de $\mathrm{C}$ orgânico do solo, mas influenciou sua distribuição vertical. Enquanto a adição de resíduos vegetais na superfície contribuiu para o acúmulo de $\mathrm{C}$ orgânico nas camadas superficiais do solo em plantio direto, a incorporação dos resíduos vegetais durante as operações de preparo resultou em maiores concentrações de C orgânico em subsuperfície no solo em preparo convencional.

\section{LITERATURA CITADA}

ANDRÉN, O.; STEEN, E. \& RAJKAI, K. Modelling the effects of moisture on barley straw and root decomposition in the field. Soil Biol. Biochem., 24:727-736, 1992.

BAYER, C. \& MIELNICZUK, J. Características químicas do solo afetadas por métodos de preparo e sistemas de cultura. R. Bras. Ci. Solo, 21:105-112, 1997.

BAYER, C.; MIELNICZUK, J.; AMADO, T.J.C.; MARTINNETO, L. \& FERNANDES, S.A. Organic matter storage in a sandy clay loam Acrisol affected by tillage and cropping systems in southern Brazil. Soil Till. Res., 54:101-109, 2000.

BERGAMASCHI, H.; GUADAGNIN, M.R.; CARDOSO, L.S. \& SILVA, M.I.G. Clima da Estação Experimental da UFRGS (e Região de Abrangência). Porto Alegre, Universidade Federal do Rio Grande do Sul, 2003. 78p.

BERGONCI, J.I.; BERGAMASCHI, H.; SANTOS, A.O.; FRANÇA, S. \& RADIN, B. Eficiência da irrigação em rendimento de grãos e matéria seca de milho. Pesq. Agropec. Bras., 36:949-956, 2001.

BLOEM, J.; RUITER, P.C.; KOOPMAN, G.J.; LEBBINK, G. \& BRUSSAARD, L. Microbial numbers and activity in dried and rewetted arable soil under integrated and conventional management. Soil Biol. Biochem., 24:655665, 1992.

CUNHA, G.R. \& BERGAMASCHI, H. Efeitos da disponibilidade hídrica sobre o rendimento das culturas. In: BERGAMASCHI, H.; BERLATO, M.A.; MATZENAUER, R.; FONTANA, D.C.; CUNHA, G.R.; SANTOS, M.L.V.; FARIAS, J.R.B. \& BARNI, N.A., eds. Agrometeorologia aplicada à irrigação. Porto Alegre, Universidade Federal do Rio Grande do Sul, 1992. p.85-97.

CHRISTENSEN, B.T. Wheat and barley straw decomposition under field conditions: Effects of soil type and plant cover on weight loss, nitrogen and potassium content. Soil Biol. Biochem., 17:691-697, 1985.

DALAL, R.C. \& MAYER, R.J. Long-term trends in fertility of soils under continuous cultivation and cereal cropping in southern Queensland. II. Total organic carbon and its rate of loss from the soil profile. Aust. J. Soil Res., 24:281$292,1986$. 
DALMAGO, G.A. Dinâmica da água no solo em cultivos de milho sob plantio direto e preparo convencional. Porto Alegre, Universidade Federal do Rio Grande do Sul, 2004. 245p. (Tese de Doutorado)

DE BONA, F.D. Dinâmica da matéria orgânica do solo em sistemas irrigados por aspersão sob plantio direto e preparo convencional. Porto Alegre, Universidade Federal do Rio Grande do Sul, 2005. 130p. (Tese de Mestrado)

ELLERT, B.H. \& BETTANY, J.R. Calculation of organic matter and nutrients stored in soils under contrasting management regimes. Can. J. Soil Sci., 75:529-538, 1995.

EMPRESA BRASILEIRA DE PESQUISA AGROPECUÁRIA EMBRAPA. Centro Nacional de Pesquisa de Solos. Sistema brasileiro de classificação de solos. Rio de Janeiro, 1999. $412 \mathrm{p}$

HÉNIN, S. \& DUPUIS, M. Essai de bilan de la matière organique du soil. Ann. Agron., 15:17-29, 1945.

LOVATO, T.; MIELNICZUK, J.; BAYER, C. \& DIEKOW, J. A method for estimating coefficients of soil organic matter dynamics based on long-term soil management experiments. Soil Till. Res., 2005. Submetido.

MANN, L.K. Changes in soil carbon storage after cultivation. Soil Sci., 142:279-288, 1986.

MERTEN, G. \& MIELNICZUK, J. Distribuição do sistema radicular e dos nutrientes em Latossolo Roxo sob dois sistemas de preparo de solo. R. Bras. Ci. Solo, 15:369-374, 1991.
MIELNICZUK, J.; BAYER, C.; VEZZANI, F.M.; LOVATO, T.; FERNANDES, F.F. \& DEBARBA, L. Manejo de solo e culturas e sua relação com os estoques de carbono e nitrogênio do solo. In: CURI, N.; MARQUES, J.J.; GUILHERME, L.R.G.; LIMA, J.M.; LOPES, A.S. \& ALVAREZ V., V.H. Tópicos em ciência do solo., eds. Viçosa, MG, Sociedade Brasileira de Ciência do Solo, 2003. v.3. p.209-248,

MITTMANN, L.M. Irrigação: Escolha o método ideal ou amargue o prejuízo. R. A Granja, 671:24-29, 2004.

ORCHARD, V.A. \& COOK, F.J. Relationship between soil respiration and soil moisture. Soil Biol. Biochem., 15:447$453,1983$.

REICOSKY, D.C.; KEMPER, W.D.; LANGADALE, G.W.; DOUGLAS, C.L. \& RASMUNSSEN, P.E. Soil organic matter changes resulting from tillage and biomass production. J. Soil Water Conserv., 50:253-261, 1995.

SAS Institute. The SAS system for Windows, Release 6.08. Cary, NC, 1996. 633p.

WIEDER, R.K. \& LANG, G.E. A critique of the analytical methods used in examining decomposition data obtained from litter bags. Ecology, 63:1636-1642, 1982.

WOODRUFF, C.M. Estimating the nitrogen delivery of soil from the organic matter determination as reflected by Sanborn field. Soil Sci. Soc. Am. Proc., 14:208-212, 1949. 